\title{
Electron Correlation in High Temperature Cuprates
}

\author{
Takashi Yanagisawa', Mitake Miyazaki², Kunihiko Yamaji ${ }^{1}$ \\ ${ }^{1}$ Electronics and Photonics Research Institute, National Institute of Advanced Industrial Science and Technology, \\ 1-1-1 Umezono, Tsukuba, Japan \\ ${ }^{2}$ Hakodate National College of Technology, 14-1 Tokura, Hakodate, Japan \\ Email: t-yanagisawa@aist.go.jp
}

Received January 2014

\begin{abstract}
Electron correlation plays a key role in high-temperature cuprate superconductors. Material-parameter dependence of cuprates is important to clarify the mechanism of high temperature superconductivity. In this study, we examine the ground state of the three-band Hubbard model (d-p model) that explicitly includes oxygen $p$ orbitals. We consider the half-filled case with the large on-site Coulomb repulsion $U_{\mathrm{d}}$ by using the variational Monte Carlo method. The ground state is insulating when $U_{\mathrm{d}}$ is large at half-filling. The ground state undergoes a transition from a metal to a Mott insulator when the level difference $\varepsilon_{\mathrm{p}}-\varepsilon_{\mathrm{d}}$ is increased.
\end{abstract}

\section{Keywords}

High-Temperature Superconductor, Electron Correlation, Mott Insulator, Metal-Insulator Transition, Charge-Transfer Insulator

\section{Introduction}

The study of high-temperature superconductors has been addressed extensively since the discovery of cuprate superconductors [1]. The $\mathrm{CuO}_{2}$ plane in cuprates plays a key role for the appearance of superconductivity [2-10] and the electron correlation in this plane is important [11-16].

Relationship between material parameters and critical temperature $T_{\mathrm{C}}$ is important to clarify the mechanism of high temperature superconductivity. We consider two kinds of material parameters. The first category includes transfer integrals $t_{\mathrm{dp}}, t_{\mathrm{pp}}$ and the level of $\mathrm{d}$ and $\mathrm{p}$ electrons. These parameters determine the band structure and the Fermi surface. The $t_{\mathrm{dp}}$ is the transfer integral between nearest $\mathrm{d}$ and $\mathrm{p}$ orbitals in the $\mathrm{CuO}_{2}$ plane, and $t_{\mathrm{pp}}$ is that between nearest $\mathrm{p}$ orbitals. The other category is concerning with the strength of interactions such as the Coulomb interaction, $U_{\mathrm{d}}$ and $U_{\mathrm{p}}$, and the electron-phonon interaction. The transfer integrals play an important role to obtain a finite bulk limit of the superconducting condensation energy $[12,13,17]$. The parameter values were estimated in the early stage of research of high temperature cuprates [18-21].

In this paper, we investigate the ground state of the three-band d-p model in the half-filled case. When the Coulomb interaction $U_{\mathrm{d}}$ is large, the ground state is presumably insulating. We show, in fact, that there is a transition from a metallic state to an insulating state as the level difference between $\mathrm{d}$ and $\mathrm{p}$ electrons is increased. 


\section{Hamiltonian}

The three-band Hamiltonian with $\mathrm{d}$ and p electrons is

$$
\begin{aligned}
& H=\varepsilon_{d} \sum_{i \sigma} d_{i \sigma}^{+} d_{i \sigma}+\varepsilon_{p} \sum_{i \sigma}\left(p_{i+\hat{x} / 2, \sigma}^{+} p_{i+\hat{x} / 2, \sigma}+p_{i+\hat{y} / 2, \sigma}^{+} p_{i+\hat{y} / 2, \sigma}\right)+t_{d p} \sum_{i \sigma}\left[d_{i \sigma}^{+}\left(p_{i+\hat{x} / 2, \sigma}+p_{i+\hat{y} / 2, \sigma}-p_{i-\hat{x} / 2, \sigma}-p_{i-\hat{y} / 2, \sigma}\right)+h . c .\right] \\
& +t_{p p} \sum_{i \sigma}\left(p_{i+\hat{y} / 2, \sigma}^{+} p_{i+\hat{x} / 2, \sigma}-p_{i+\hat{y} / 2, \sigma}^{+} p_{i-\hat{x} / 2, \sigma}-p_{i-\hat{y} / 2, \sigma}^{+} p_{i+\hat{x} / 2, \sigma}+p_{i-\hat{y} / 2, \sigma}^{+} p_{i-\hat{x} / 2, \sigma}+\text { h.c. }\right)+t_{d}^{\prime} \sum_{\langle i j\rangle \sigma}\left(d_{i \sigma}^{+} d_{j \sigma}+\text { h.c. }\right) \\
& +U_{d} \sum_{i} n_{i \uparrow}^{d} n_{i \downarrow}^{d}+U_{p} \sum_{i}\left(n_{i+\hat{x} / 2 \uparrow}^{p} n_{i+\hat{x} / 2 \downarrow}^{p}+n_{i+\hat{y} / 2 \uparrow}^{p} n_{i+\hat{y} / 2 \downarrow}^{p}\right)
\end{aligned}
$$

where $U_{\mathrm{d}}$ and $U_{\mathrm{p}}$ indicate the on-site Coulomb interaction among $\mathrm{d}$ and p electrons, respectively. $d_{i \sigma}$ and $d_{i \sigma}^{+}$ are operators for the $\mathrm{d}$ electrons. $p_{i \pm \hat{x} / 2, \sigma}$ and $p_{i \pm \bar{x} / 2, \sigma}^{+}$denote operators for the p electrons at the site $R_{i \pm \hat{x} / 2}$, and in a similar way $p_{i \pm \hat{y} / 2, \sigma}$ and $p_{i \pm \bar{y} / 2, \sigma}^{+}$are defined. $n_{i \sigma}^{d}$ and $n_{i+\hat{\mu} / 2, \sigma}^{p}(\mu=x, y)$ are number operators for $d$ and p electrons, respectively. We have introduced the parameter $t^{\prime}{ }_{\mathrm{d}}$ that is the transfer integral of $\mathrm{d}$ electrons between next nearest-neighbor cooper sites, where <ij>denotes a next nearest-neighbor pair of copper sites. The energy unit is given by $t_{\mathrm{dp}}$ in this paper. We use the notation $\boldsymbol{\Delta}_{\mathbf{d p}}=\varepsilon_{\mathrm{p}}-\varepsilon_{\mathrm{d}}$. The number of sites is denoted as $N_{\mathrm{s}}$, and the total number of atoms is denoted as $N_{\mathrm{a}}=3 N_{\mathrm{s}}$. Our study is within the hole picture where the lowest band is occupied up to the Fermi energy $\mu$. The non-interacting part is written as

$$
H_{0}=\sum_{k \sigma}\left(d_{k \sigma}^{+} p_{x k \sigma}^{+} p_{y k \sigma}^{+}\right)\left(\begin{array}{ccc} 
& & \\
\varepsilon_{d k}-\mu & \varepsilon_{x k} & \varepsilon_{y k} \\
-\varepsilon_{x k} & \varepsilon_{p}-\mu & \varepsilon_{p k} \\
-\varepsilon_{y k} & \varepsilon_{p k} & \varepsilon_{p}-\mu
\end{array}\right)\left(\begin{array}{l}
d_{k \sigma} \\
p_{x k \sigma} \\
p_{y k \sigma}
\end{array}\right)
$$

where $\varepsilon_{\mathrm{xk}}=2 i t_{\mathrm{dp}} \sin \left(k_{\mathrm{x}} / 2\right), \varepsilon_{\mathrm{yk}}=2 i t_{\mathrm{dp}} \sin \left(k_{\mathrm{y}} / 2\right), \varepsilon_{\mathrm{pk}}=-4 t_{\mathrm{pp}} \sin \left(k_{\mathrm{x}} / 2\right) \sin \left(k_{\mathrm{y}} / 2\right)$ and $\varepsilon_{\mathrm{dk}}=-4 t_{\mathrm{d}} \cos \left(k_{\mathrm{x}}\right) \cos \left(k_{\mathrm{y}}\right) \cdot p \mu_{\mathrm{k \sigma}}$ and $d_{k \sigma}$, are Fourier transforms of $p_{i+\tilde{\mu} / 2, \sigma}$ and $d_{i \sigma}$, respectively. The eigenvectors of this matrix give the corresponding weights of $\mathrm{d}$ and p electrons.

\section{Mott State and Wave Function}

\subsection{Gutzwiller Function}

We adopt the Gutzwiller ansatz for the wave function:

$$
\psi_{G}=P_{G}^{d} \psi_{0}
$$

where $P_{\mathrm{G}}$ is the Gutzwiller projection operator given by

$$
P_{G}^{d}=\prod_{i}\left[1-(1-g) n_{i \uparrow}^{d} n_{i \downarrow}^{d}\right]
$$

with the variational parameter in the range from 0 to unity: $0 \leq \mathrm{g} \leq 1$. The operator $P_{\mathrm{G}}$ controls the on-site electron correlation on the copper site. When we take into account $U_{\mathrm{p}}$, the correlation among p electrons is also considered. In this case $P_{\mathrm{G}}$ is

where

$$
P_{G}=P_{G}^{d} P_{G}^{p}
$$

$$
P_{G}^{p}=\prod_{i}\left[1-\left(1-g_{p}\right) n_{i+\hat{x} / 2 \uparrow}^{p} n_{i+\hat{x} / 2 \downarrow}^{p}\right]\left[1-\left(1-g_{p}\right) n_{i+\hat{y} / 2 \uparrow}^{p} n_{i+\hat{y} / 2 \downarrow}^{p}\right]
$$

with the parameter $g_{\mathrm{p}}$ in the range $0 \leq g_{\mathrm{p}} \leq 1 . \psi_{0}$ is a one-particle wave function. We can take various kinds of states for $\psi_{0}$; for example, the Fermi sea or the Hartree-Fock state with some order parameters.

\subsection{Optimized Wave Function}

There are several ways to improve the Gutzwiller function. One method is to consider an optimization operator: 


$$
\psi=\exp (\lambda K) \psi_{G}
$$

where $K$ is the kinetic part of the total Hamiltonian $H$ and $\lambda$ is a variational parameter [9]. The ground state energy is lowered appreciably by the introduction of $\lambda$ [11]. This type of wave function is an approximation to the wave function in quantum Monte Carlo method [22-24].

We note that the Gutzwiller function $\psi_{\mathrm{G}}$ cannot describe an insulating state at half-filling because we have no kinetic energy gain in the limit $g \rightarrow 0$. A wave function for the Mott state has been proposed for the single-band Hubbard model by adopting the doublon-holon correlation factor [25]. In this paper, instead, we consider the optimized Gutzwiller function in Equation (7) in the limit $\mathrm{g} \rightarrow 0$ as a Mott insulating state. This is an insulator of charge-transfer type [26] and is a metal-insulator transition in a multi-band system [27].

\subsection{Mott State in the Single-Band Case}

Here we examine the Mott insulating state for the single-band Hubbard model [28]. We show the ground-state energy per site as a function of $U$ in Figure 1, obtained by using the wave function in Equation (7). The curvature of the energy, as a function of $U$, changes near $U \sim 8$ and the parameter $g$ vanishes simultaneously. The state with vanishing $g$ would be an insulating state because of vanishingly small double occupancy.

\subsection{Variational Parameters of the Band Structure}

In the three-band case, we have additional band parameters as variational parameters in $\psi_{0}$. The one-particle state $\psi_{0}$ contains the variational parameters $\tilde{t}_{d p}, \tilde{t}_{p p}, \tilde{t}^{\prime}{ }_{d}, \tilde{\varepsilon}_{d}$ and $\tilde{\varepsilon}_{p}$ :

$$
\psi_{0}=\psi_{0}\left(\tilde{t}_{d p}, \tilde{t}_{p p}, \tilde{t}_{d}^{\prime}, \tilde{\varepsilon}_{d}, \tilde{\varepsilon}_{p}\right)
$$

In the non-interacting case, $\tilde{t}_{d p}, \tilde{t}_{p p}$ and $\tilde{t}^{\prime}{ }_{d}$ coincide with $t_{\mathrm{dp}}, t_{\mathrm{pp}}$ and $t_{\mathrm{d}}{ }_{\mathrm{d}}$, respectively. The expectation values of physical quantities are calculated by employing the variational Monte Carlo method [6,7].

\section{Mott State of Charge-Transfer Type}

Our study on the Mott state of the three-band model is based on the wave function in Equation (7). The ground-state energy per site $E / \mathrm{N}_{\mathrm{s}}-\varepsilon_{\mathbf{d}}$ as a function of the level difference $\Delta_{\mathrm{dp}}$ is shown in Figure 2. The parameters are $\mathrm{t}_{\mathbf{p p}}=0.4, \mathrm{t}_{\mathbf{d}}=0.0$ and $U_{\mathbf{d}}=8$. We set $U_{\mathbf{p}}=0$ for simplicity because $U_{\mathbf{p}}$ is not important in the low doping case and also in the half-filled case. The parameter $\mathrm{g}$ for the optimized function $\psi$ vanishes at $\Delta_{\mathbf{d p}} \approx 2$ while that for the Gutzwiller function $\psi_{\mathrm{G}}$ remains finite even for large $\Delta_{\mathrm{dp}}$. The result shows that there is a transition from a metallic state to an insulating state at the critical value of $\Delta_{\mathrm{dp}} \sim \approx\left(\Delta_{\mathrm{dp}}\right)_{\mathrm{c}} \sim 2$.

We find that the curvature of the energy, as a function of $\Delta_{\mathrm{dp}}$, is changed near $\Delta_{\mathrm{dp}} \sim 2$. The energy is well fit-

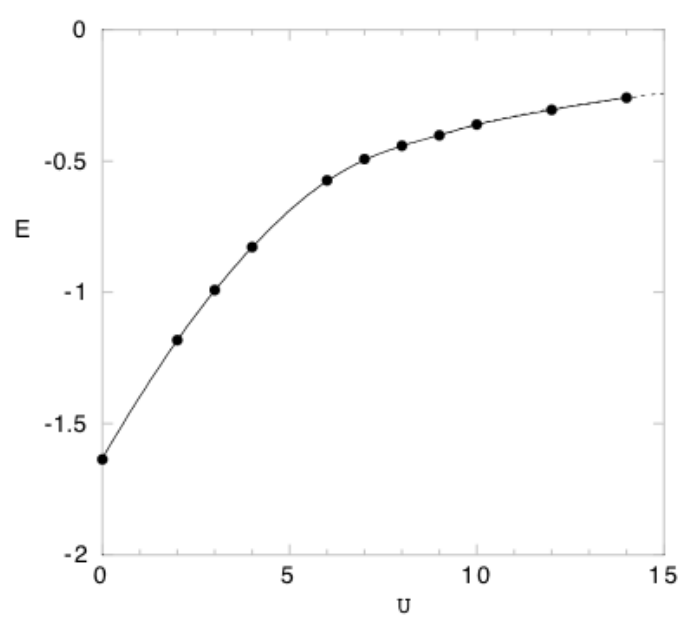

Figure 1. Ground state energy of the 2D singleband Hubbard model as a function of $U$ at half-filling. The system size is $6 \times 6$. 


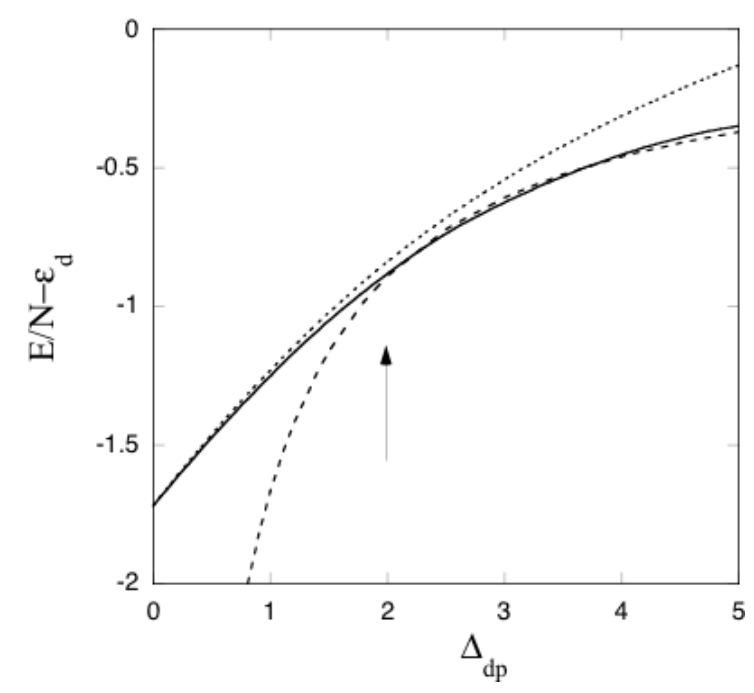

Figure 2. Ground-state energy of the 2D d-p model per site as a function of $\Delta_{\mathrm{dp}}$ for $\mathrm{t}_{\mathrm{pp}}=0.4, \mathrm{t}_{\mathrm{d}}=0.0$ and $U_{d}=8$ (in units of $t_{d p}$ ) in the half-filled case on $6 \times 6$ lattice. The arrow indicates a transition point where the curvature is changed. The dotted curve is for the Gutzwiller function $\psi_{\mathrm{G}}$ (with $\lambda=0$ ). The dashed curve indicates that given by a constant times $1 /\left(\boldsymbol{\varepsilon}_{\mathrm{p}}-\boldsymbol{\varepsilon}_{\mathrm{d}}\right)$.

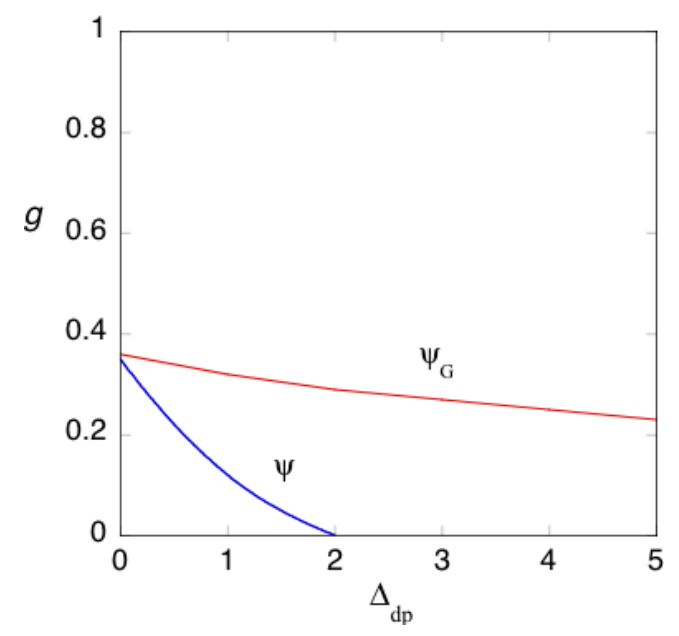

Figure 3. The Gutzwiller parameter $g$ as a function of $\Delta_{\mathrm{dp}}$ for $\psi_{\mathrm{G}}$ and the optimized wave function $\psi \cdot g$ for $\psi$ decreases and vanishes as $\Delta_{\mathrm{dp}}$ is increased.

ted by $1 / \Delta_{\mathrm{dp}}$ when $\Delta_{\mathrm{dp}}$ is greater than $\left(\Delta_{\mathrm{dp}}\right)_{\mathrm{c}}$. This is shown in Figure 2 where the dashed curve indicates $1 / \Delta_{\mathrm{dp}}$. This shows that the most of energy gain comes from the exchange interaction between nearest neighbor $d$ and $p$ electrons. This exchange interaction is given by $\mathrm{J}_{\mathrm{K}}$ :

$$
J_{K}=t_{d p}^{2}\left(\frac{1}{\Delta_{d p}}+\frac{1}{U_{d}-\Delta_{d p}}\right)
$$

In the insulating state, the energy gain is proportional to $\mathrm{J}_{\mathrm{K}}$, which is consistent with our result.

We show the Gutawiller parameter $g$ as a function of the level difference $\Delta_{\mathrm{dp}}$ in Figure 3. $\mathrm{g}$ for the Gutzwiller 
function $\psi_{\mathrm{G}}$ descreases gradually when $\Delta_{\mathrm{dp}}$ is increased. In contrast, $g$ for the optimized function $\psi$ shows a rapid decrease and almost vanishes near $\left(\Delta_{\mathrm{dp}}\right)_{\mathrm{c}}$. This is consistent with the behavior of the energy shown in Figure 2, indicating that the ground state is an insulator when $\Delta_{\mathrm{dp}}>\left(\Delta_{\mathrm{dp}}\right)_{\mathrm{c}}$. When $g$ vanishes, the double occupancy of $\mathrm{d}$ holes is completely excluded and we have exactly one hole on the copper site. This is the insulating state of charge-transfer type.

\section{Summary}

We have investigated the ground state of the three-band d-p model at half-filling by using the variational Monte Carlo method. We have proposed the wave function for an insulating state of charge-transfer type with an optimization operator on the basis of the Gutzwiller wave function. We have shown that this wave function describes a transition from a metallic state to an insulating state as the level difference $\Delta_{\mathrm{dp}}$ is increased. The critical value of $\Delta_{\mathrm{dp}}$ would depend on $U_{\mathrm{d}}$ and band parameters.

\section{Acknowledgements}

We thank J. Kondo and I. Hase for useful discussions.

\section{References}

[1] Bennemann, K.H. and Ketterson, J.B. (2003) The Physics of Superconductors Vol. 1 and Vol. II. Springer-Verlag, Berlin.

[2] Hirsch, J.E., Loh, D., Scalapio, D.J. and Tang, S. (1989) Physical Review, B39, 243.

[3] Scalettar, R.T., Scalapino, D.J., Sugar, R.L. and White, S.R. (1989) Physical Review, B39, 243.

[4] Guerrero, M., Gubernatis, J.E. and Zhang, S. (1998) Physical Review, B57, 11980.

[5] Koikegami, S. and Yamada, K. (2000) Journal of the Physical Society of Japan, 69, 768.

[6] Yanagisawa, T., Koike, S. and Yamaji, K. (2001) Physical Review, B64, 184509.

[7] Yanagisawa, T., Koike, S. and Yamaji, K. (2003) Physical Review, B67, 132408.

[8] Weber, C., Lauchi, A., Mila, F. and Giamarchi, T. (2009) Physical Review Letters, 102, 017005.

[9] Yanagisawa, T., Miyazaki, M. and Yamaji, K. (2009) Journal of the Physical Society of Japan, 78, 013706.

[10] Lau, B., Berciu, M. and Sawatzky, G.A. (2011) Physical Review Letters, 106, 036401.

[11] Yanagisawa, T., Koike, S. and Yamaji, K.( 1998) Journal of the Physical Society of Japan, 67, 3867.

[12] Koike, S., Yamaji, K. and Yanagisawa, T. (1999) Journal of the Physical Society of Japan, 68, 1657.

[13] Yamaji, K., Yanagisawa, T. and Koike, S. (2000) Physica, B284-288, 415.

[14] Yamaji, K., Shimoi, Y. and Yanagisawa, T. (1994) Physica, C235-240, 2221.

[15] Miyazaki, M., Yanagisawa, T. and Yamaji, K. (2004) Journal of the Physical Society of Japan, 73, 1643.

[16] Miyazaki, M., Yamaji, K., Yanagisawa, T. and Kadono, R. (2009) Journal of the Physical Society of Japan, 78, 043706.

[17] Yamaji, K. and Yanagisawa, T. (2010) Journal of the Physical Society of Japan, 80, 083702.

[18] Hybertsen, M.S., Schluter, M. and Christensen, N.E. (1989) Physical Review, B39, 9028.

[19] Eskes, H., Sawatzky, G.A. and Feiner, L.F. (1989) Physica, C160, 424.

[20] McMahan, A.K., Annett, J.F. and Martin, R.M. (1990) Physical Review, B42, 6268.

[21] Eskes, H. and Sawatzky, G.A. (1991) Physical Review, B43, 119.

[22] Hirsch, J.E., Scalapino, D.J. and Sugar, R.L. (1981) Physical Review Letters, 47, 1628.

[23] Yanagisawa, T. (2007) Physical Review, B75, 224503.

[24] Yanagisawa, T. (2013) New Journal of Physics, 15, 033012.

[25] Yokoyama, H., Ogata, M. and Tanaka, Y. (2006) Journal of the Physical Society of Japan, 75, 114706.

[26] Zaanen, J., Sawatzky, G.A. and Allen, J.W. (1985) Physical Review Letters, 55, 418.

[27] Blawid, S., Tuan, H.A., Yanagisawa, T. and Fulde, P. (1996) Physical Review, B54, 7771.

[28] Hubbard, J. (1963) Proceedings of the Royal Society, A276, 237. 